\title{
Self-regulation as a Mediator of Mindfulness and Physical Activity: A Narrative Review
}

\author{
Aaron Boyle, Amber Mosewich
}

Faculty of Kinesiology, Sport, and Recreation, University of Alberta, Edmonton, Alberta

Corresponding author: aboyle@ualberta.ca

\section{ABSTRACT}

Mindfulness is gaining attention as a means of increasing physical activity (PA) participation. Given that only $15.4 \%$ of adult Canadians currently meet the Canadian Physical Activity Guidelines (Colley et al., 2011), it is imperative to find ways to increase PA among adults. One way to accomplish this is to promote self-regulation skills since self-regulation is among the top predictors of PA participation (Teixeira et al., 2015). The purpose of this narrative review was to further understand the role of self-regulation as a potential mechanism through which mindfulness may impact PA participation. Initially, 160 papers were identified by title for this review. After reading abstracts, 37 papers were identified as possibly relating to the topic of interest. Following full readings, 26 papers were included in the final review. Due to the novelty of this topic, there is limited research on the mechanisms by which mindfulness may be related to physical activity. Review of the literature suggests that self-regulation appears to be a promising mechanism by which mindfulness could improve physical activity participation (Shapiro et al., 2006; Samdal et al., 2017), as self-regulation has been shown to play an important role in behaviour change. However, other alternative mechanisms include improved self-efficacy, as well as improved satisfaction (Neace et al., 2020; Tsafou et al., 2016). The paper concludes that more research on the mechanisms of mindfulness on PA, specifically self-regulation as a mechanism, could foster more knowledgeable intervention practices, and consequently improve mindfulness-based interventions efficacy.

\section{Introduction}

Physical activity (PA), which is defined as any bodily movement produced by skeletal muscles that results in energy expenditure (Caspersen et al.,1985), has widely been accepted as an integral part of healthy living (Penedo \& Dahn, 2005; Warburton et al., 2006). Benefits of engaging in regular PA include a lowered risk of cardiovascular disease and allcause mortality (Lollgen et al., 2009; Warburton et al., 2006), as well as a reduction in depression and anxiety (Rebar et al., 2015). Despite these benefits, participation in consistent PA is still low among the general adult population worldwide and in Canada (WHO, 2010; Health Status of Canadians 2016). In response to the low levels of PA, there has been a variety of behavioural interventions aimed at trying to increase PA, with most having only moderate success (Conn et al., 2011; Denison et al., 2014). As such, new behavioural interventions are being developed

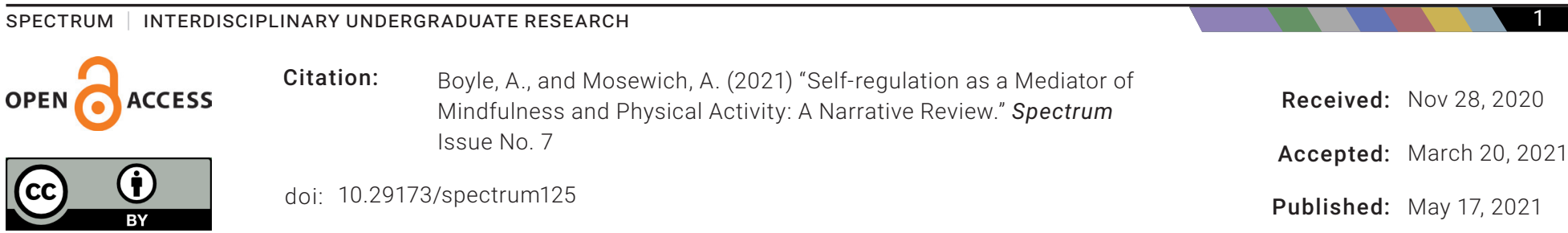


in an effort to stop the rise in sedentary time and increase PA participation.

One construct that has recently gained attention in the behaviour change literature is mindfulness. Kabat-Zinn's definition of mindfulness that will be used in this paper is "the awareness that emerges through paying attention on purpose, in the present moment, and nonjudgmentally, to the unfolding of experience moment by moment" (Kabat-Zinn, 2003, p 145). Mindfulness is both a character trait that differs naturally between individuals that is generally stable across time (Schneider et al., 2019; Yang \& Conroy, 2019), as well as a state or moment of awareness that may shift from one moment to the next regardless of an individual's level of trait mindfulness (Yang \& Conroy, 2019). Therefore, mindfulness is conceptualized both as an inherent trait as well as a skill that can be trained and improved upon. Some common forms of mindfulness training/mindfulness-based interventions (MBI's) include mindfulness meditation, mindfulness-based stress reduction (MBSR), mindfulness-based cognitive therapy (MBCT), dialectical behaviour therapy (DBT), and acceptance and commitment therapy (ACT) (Keng et al., 2011). Mindfulness training has been used in different contexts and has shown a wide range of positive results ranging from management of chronic pain (Chiesa \& Serretti, 2011), to uses in education (Felver et al., 2016) and psychology (Keng et al., 2011).

With the benefits of being mindful becoming increasingly clear, sports psychologists, as well as epidemiologists, have begun researching its uses in sport, exercise, and physical activity. Despite efforts made towards understanding mindfulness and its role in PA, there is still much that is unknown about the possible benefits of mindfulness on PA and the mechanisms by which mindfulness may act (Schneider et al., 2019). Preliminary evidence does suggest mindfulness may be associated with PA, however, evidence is still not definitive (Schneider et al., 2019). Further research must be done on the possible mechanisms by which mindfulness could influence PA participation. A clear understanding of the mechanisms will provide insight into how best to design and implement mindfulness interventions that are effective in increasing PA engagement, and also will provide useful knowledge towards understanding mindfulness as a whole.

One mechanism worth exploring in the mindfulness and PA participation relationship is self-regulation. Self-regulation refers to "a broad construct representing the cognitive, motivational-affective, social and physiological processes that modulate attention, emotion and behaviour to a given situation/stimulus, for the purpose of pursuing a goal" (Bassett et al., 2012 , p. 597). In other words, self-regulation is the ability to regulate yourself and choose actions that lead toward positive outcomes. This facilitates greater discipline and promotes goal achievement (Duckworth et al., 2011). Self-regulation has been proposed to be connected to mindfulness as a mechanism through which mindfulness acts to bring about behaviour change (Shapiro et al., 2006). Self-regulation has also been shown to be associated with PA behaviour change (Rhodes \& Pfaeffli, 2010). Therefore, the goal of this paper is to perform a narrative review of the relationship between mindfulness, PA participation, and self-regulation and to explore self-regulation as a possible mechanism through which mindfulness could influence PA participation.

\section{Mindfulness and PA}

In recent years, several review papers have been published that overview mindfulness and PA participation (Schneider et al., 2019; Yang \& Conroy, 2019). As such, this section will briefly cover what was found by Schneider and Yang, and then review the research that has been published since Schneider et al. (2019) in order to perform a narrative review of the research on mindfulness and PA up until June 2020. Schneider et al. (2019) reviewed the role of dispositional (trait) mindfulness, mindfulness-based interventions, and the mechanisms by which mindfulness may work, finding forty papers related to mindfulness and PA participation. "Dispositional mindfulness is defined as a basic human quality which everyone possesses 
to a varying extent" and is enduring across time and context (Schneider et al., 2019; Yang \& Conroy, 2019). Twenty papers looked at the relationship of dispositional mindfulness and PA engagement with nine out of twenty papers finding a positive correlation with PA engagement. Twenty papers looked at the effectiveness of MBIs, which seek to teach participants mindful behaviours that can be applied to their daily lives. Fifteen of the twenty papers investigated by Schneider (2019) showed positive associations between MBIs and PA. This shows the effectiveness of mindfulness training, illustrating that mindfulness is a quality that can be learned.

Schneider (2019) also looked at potential mechanisms, finding five papers showing satisfaction, lowered stress, and psychological flexibility as possible mechanisms by which mindfulness affects PA. In their 2016 study and 2017 follow-up study, Tsafou and colleagues suggested that mindfulness may increase awareness of the positive experiences of $P A$, resulting in greater feelings of satisfaction and increased PA engagement and adherence. Since satisfaction with the activity is predicted to influence behaviour change and maintenance (Rothman et al., 2004), satisfaction could be a potential mechanism by which mindfulness influences PA engagement.

In a 2010 study, Roberts and Danoff-Burg found increased mindfulness was related to lowered stress levels, which led to greater engagement in positive health behaviour like PA (2010). Psychological flexibility was proposed as one of the ways through which mindfulness may reduce stress. Mindfulness demands an awareness to the present which facilitates openness to different possibilities. Therefore, mindfulness seems to be connected with greater psychological flexibility, which results in decreased stress, which may increase PA levels (Sagui-Henson et al., 2018). Schneider and colleagues (2019) concluded that there is preliminary evidence to suggest $\mathrm{PA}$ is associated with mindfulness; however, more research needs to occur, specifically more research needs to be done on the mechanisms through which mindfulness acts on PA.
Yang \& Conroy (2019) also performed a systematic review that identified 13 papers looking at trait (i.e., dispositional) and state mindfulness. State mindfulness, in contrast to trait mindfulness, varies within people across time and contexts regardless of the person's level of trait mindfulness (Yang \& Conroy, 2019). Ten of the thirteen papers discussed showed a positive relation between mindfulness and PA. Of particular note is the lack of research on state mindfulness and PA, as only three papers identified by Yang et al. addressed state mindfulness and PA.

In the review of the literature since Schneider et al. (2019), five papers were identified, not including Yang (2019), that looked at the association between mindfulness and PA, including a review paper and meta-analysis (Lyzwinski et al., 2018; Sala et al., 2019), two cross-sectional studies (Lentz \& Brown, 2019; Strowger et al., 2018), and one randomized control trial (Robin et al., 2020). Both the review and meta-analysis found a positive relationship between PA and trait mindfulness although both primarily focused on health behaviors as a whole. The two cross-sectional studies also showed a positive relationship with mindfulness and PA. Of particular interest is the Strowger (2018) study which had a sample size of over 30,000 people. The study concluded that mindfulness meditation was associated with less sedentary time and a greater likelihood to meet the aerobic activity recommendations as put forward by the WHO guidelines. The randomized control trial looked at the effect of text messages reminding participants to do their ten-minute mindful meditation every day over a two-week period. They found those who engaged in more mindful meditation had significantly higher weekly values of aerobic activity when compared to the control group (Robin et al., 2020). These studies, when taken together with the Schneider (2019) and Yang (2019) reviews, show promising evidence that mindfulness could be an effective tool to help increase PA participation. 


\section{Mindfulness and Self-regulation}

Self-regulation has consistently been connected with positive behaviour change (Miller et al., 2020), particularly in the realm of PA engagement (Rhodes \& Pfaeffli, 2010). In Shapiro's 2006 paper it was proposed that self-regulation was one possible way through which mindfulness could act in order to achieve behaviour change (Shapiro et al., 2006). Self-regulation is typically broken into two main parts: emotional regulation and executive functioning. Emotional regulation refers to the process by which one monitors, evaluates, and modifies their emotions in an effort to be more in control of their emotions (Artino, 2011). Emotional regulation is especially important in goal directed behaviour, as a lack of it can lead to automaticity of habits and a consequent lack of behavioural change (Chambers et al., 2009). Being mindful can assist with emotional regulation by bringing awareness to the present moment, and non-judgmentally reacting to any negative emotions or cravings one may have, decreasing automaticity. Executive functioning is broken down further into three main functions: working memory, response inhibition, and psychological flexibility. When relating mindfulness to the executive functions, it is believed that mindfulness is closely linked to response inhibition and working memory (Casedas et al., 2019). This makes sense because mindfulness, with the goal of paying attention to the present moment, would likely foster a mind that is concentrated. This concentration would contribute to an increased working memory, as well as response inhibition of other distracting experiences. These abilities in turn foster greater self-regulation skills and presumably increase the chance of behaviour change.

The positive relationship between mindfulness and self-regulation was further confirmed through brain imaging techniques. The hippocampus - which is responsible for processes such as learning, memory consolidation, and self-regulation - was studied in an eight-week mindfulness training intervention. This study found a significant increase in gray matter in the hippocampus following the eight weeks of mind- fulness training (Hölzel et al., 2011). Gray matter at its most basic level is a collection of neurons. It is found throughout the brain and spinal cord and is essential to body function. An increase in gray matter in any region of the brain is associated with an increased performance in the functions of that particular area of the brain (Hölzel et al., 2011). Therefore, an increase in gray matter of the hippocampus is an anatomical indicator of its increased ability to perform its key functions - one of which is self-regulation. Another study by Tang and colleagues recruited participants for a ten-year mindfulness training intervention. Their results showed greater brain connectivity in the areas of the brain associated with self-regulation. From this, the authors concluded that long-term mindfulness meditation training results in enhanced self-regulation (Tang et al., 2020). The longitudinal design of this study adds robust evidence for the association between mindfulness training and self-regulation. Self-regulation, then, seems to have an important role to play in the way mindfulness may influence behaviour. Therefore, it is imperative to further address self-regulation as a potential mechanism for PA engagement.

\section{Self-regulation and PA}

Having established that mindfulness appears to be connected to self-regulation, the relationship between PA participation and self-regulation will be discussed next. There is extensive literature on the role self-regulation plays in PA behaviour, so this is only a narrative review of the work done. A 2000 study found a positive relationship between self-management techniques (goal setting, self-monitoring) and physical activity among college-aged women (Saelens et al., 2000). This study paved the way for future research in the area of self-regulation and PA. In another study in 2012, it was found that self-regulation mediated the intention-behaviour gap in exercise, indicating self-regulation may be essential for converting intentions into behaviour (de Bruin et al., 2012). The intention-behaviour gap is one of the biggest conundrums in psychology. With many people aware of the benefits of PA and intending to participate, but many 
still not engaging in PA, it would seem that bridging this gap is essential for PA behaviour change. A 2011 study discovered that self-regulation was associated with increased self-efficacy, which helped predict physical activity adherence (McAuley et al., 2011). The McAuley study suggests that self-regulation can assist with starting a goal (such as starting an exercise program) and the maintenance and consistency of working towards that goal. Both behaviours are key for PA participation. In line with this assertion, a 2011 review identified self-regulation skills as an important factor in health behaviour change and maintenance (Greaves et al., 2011). This was confirmed in 2017 by a meta-analysis and review which concluded that self-regulation skills are essential to behaviour change in PA (Samdal et al., 2017). Finally, a 2015 review found that self-regulation skills are a key mechanism by which PA outcomes could occur (Teixeira et al., 2015). These three reviews point towards self-regulation as an important part of any behaviour change and maintenance, and furthermore show that in the case of PA engagement, self-regulation plays an important role.

\section{Mindfulness, Self-regulation, and PA}

Given that self-regulation has been shown to be such a major part of mindfulness and PA, it is curious that there is a lack of literature on self-regulation as a mediator between mindfulness and PA. The studies to date that have looked at mechanisms connecting mindfulness and PA are limited. One study looked at the mediating role of satisfaction on mindfulness and PA, finding satisfaction to mediate the relationship (Tsafou et al., 2016). This is interesting, as satisfaction may play a role in self-regulatory strategies. For example, satisfaction with one's ability to achieve their goals would further induce self-management strategies that promote goal-directed behaviour. A follow up study found that trait mindfulness only was related to satisfaction through state mindfulness (De Ridder et al., 2017). This finding highlights the need to integrate state and trait mindfulness research in future studies, and not merely focus on one or the other as is common in the literature. Additionally, this finding shows the importance of state mindfulness - a vastly under researched topic in the mindfulness literature. In a 2020 study, it was found that engaging in audio-guided mindfulness exercises in a single session of self-paced walking led to increased attention toward the task and increased positive affective response following the activity (Bigliassi et al., 2020). Relating to Tsafou's study, mindfulness could enhance PA behavioural change through increased satisfaction and a decreased negative perception of the task. This recent study is a step forward in the literature regarding state mindfulness and PA research. Four other studies were identified that sought to find possible mechanisms by which mindfulness affects PA levels. Two of these studies identified stress as a possible mediator in which dispositional mindfulness leads to decreased overall stress and increased health behaviors (Roberts \& Danoff-Burg, 2010; Sagui-Henson et al. 2018). One paper by Kang and colleagues predicted that mindfulness worked through positive affect, particularly in response to health messages (2017). Another set of authors showed that mindfulness independently predicted exercise self-efficacy, concluding that mindfulness may increase PA through increased self-efficacy (Neace et al., 2020) - a well-established mechanism for PA behaviour change (Sniehotta et al., 2005).

Given the amount of literature that supports mindfulness' connection to self-regulation strategies and self-regulation strategies to PA, it is prudent that this be explored as a mechanism by which mindfulness could enhance PA. By showing such a mediation, future interventions could focus mindfulness training on self-regulation strategies in order to effectively bring about behaviour change. Practical examples of such an intervention could be consciously bringing to attention and focusing on one's goals, and the means by which they will be achieved, or it could be practicing self-monitoring strategies while learning how to be aware and in the present moment. Furthermore, mindfulness has been shown to be related to a number of other constructs such as self-efficacy, satisfaction, and decreased stress, all of which have been dis- 
cussed earlier as mechanisms by which mindfulness could affect PA engagement. Mindfulness serves as a powerful tool in which it can act not only through self-regulation strategies, but through other skills which promote PA. The fact that mindfulness works independently through many different aspects could serve to amplify its impact on behaviour change. Given the benefits of mindfulness, a further pursuit of understanding how mindfulness acts on PA will only serve to enhance the literature and offer practical strategies for PA behaviour change.

\section{Limitations}

This review has four key limitations. First, a narrative review was undertaken. While providing a thorough overview of the literature on self-regulation, mindfulness, and PA, it is not as rigorous as a systematic review or meta-analysis. This limits the validity of the review as certain important papers may have been overlooked, as much as we have sought to highlight key articles. Second, this review only included papers that could be accessed online through Google Scholar or through the University of Alberta's Access Database. This limitation means that non-digitized texts, as well as journals not supported by the University, would be excluded. Exclusion criteria also included being in English, working on humans, and being a peer reviewed article. Third, there were positive associations between the three variables shown, but the direction of cause and effect has not been firmly established in the literature. For example, mindfulness is positively associated with PA participation. Although it is hypothesized that more mindfulness leads to greater PA, it is possible more PA leads to more mindfulness. Although not established in the literature, it is entirely possible that both directions of cause and effect are true to a certain extent. Finally, it is recognized that both mindfulness and self-regulation have varying theories and operationalizations. This paper did not focus on one theory or operationalization of either construct (e.g., one-dimensional vs multidimensional mindfulness) and thus limits the interpretation of this paper through any one lens or theory. Having different operationalizations and theories may also limit the validity of the findings as comparing papers based on different presumptions can prove difficult and may not accurately represent an author's point of view.

\section{Conclusion and Future Directions}

Despite these limitations, this narrative review highlights the need for more research on the mechanisms of mindfulness in order to create more effective interventions since applications from this research could lead to increased PA participation. In particular, self-regulation should be explored through randomized control trials in order to confirm the role of self-regulation in mindfulness and PA engagement. Furthermore, research focusing on the relationship between state and trait mindfulness on $\mathrm{PA}$, and the role each one plays in behaviour change and self-regulation strategies is an important next step in mindfulness and PA research. Such research would enhance our understanding of the interaction between both trait and state mindfulness and allow for more tailored intervention strategies. Finally, although the focus of this paper was on PA participation in general, further research into the association between mindfulness and different aspects of PA (differing intensities, durations, modalities) would be of great benefit to the literature. Mindfulness has been shown to be an effective tool for behaviour change, making mindfulness training a viable, easy, and impactful solution for greater PA engagement. 


\section{Works Cited}

Artino, A.R. (2011). Regulation of Emotion. In: Goldstein S., Naglieri J.A. (Eds.), Encyclopedia of Child Behavior and Development. https://doi.org/10.1007/978-0-387-79061-9_2388

Bassett, H. H., Denham, S., Wyatt, T. M., \& Warren-Khot, H. (2012). Refining the preschool self-regulation assessment for use in preschool classrooms. Infant and Child Development, 21(6), 596-616. https:// doi.org/10.1002/icd.1763

Casedas, L., Pirruccio, V., Vadillo, M. A., \& Lupianez, J. (2019). Does mindfulness meditation training enhance executive control? A systematic review and meta-analysis of randomized controlled trials in adults. Mindfulness, 11, 411-424. https://doi.org/10.1007/s12671-019-01279-4

Caspersen, C. J., Powell, K. E., \& Christenson, G. M. (1985). Physical activity, exercise, and physical fitness: Definitions and distinctions for health-related research. Public Health Rep, 100(2), 126-131. https:// www.jstor.org/stable/20056429

Chambers, R., Gullone, E., \& Allen, N. B. (2009). Mindful emotion regulation: An integrative review. Clinical Psychology Review, 29(6), 560-572. https://doi.org/10.1016/j.cpr.2009.06.005

Chiesa, A. \& Serretti, A. (2011). Mindfulness-based interventions for chronic pain: A systematic review of the evidence. The Journal of Alternative and Complementary Medicine, 17(1), 83-93. https://doi. org/10.1089/acm.2009.0546

Conn, V. S., Hafdahl, A. R., \& Mehr, D. R. (2011). Interventions to increase physical activity among healthy adults: Meta-analysis of outcomes. American Journal of Public Health, 101(4), 751-758. https://doi. org/10.2105/AJPH.2010.194381

de Bruin, M., Sheeran, P., Kok, G., Hiemstra, A., Prins, J. M., Hospers, H. J., \& van Breukelen, G. J. (2012). Self-regulatory processes mediate the intention-behavior relation for adherence and exercise behaviors. Health Psychology, 31(6), 695. https://doi.org/10.1037/a0027425

Denison, E., Vist, G. E., Underland, V., \& Berg, R. C. (2014). Interventions aimed at increasing the level of physical activity by including organised follow-up: A systematic review of effect. BMC Family Practice, 15(1), 120. https://doi.org/10.1186/1471-2296-15-120

Duckworth, A. L., Grant, H., Loew, B., Oettingen, G., \& Gollwitzer, P. M. (2011). Self-regulation strategies improve self-discipline in adolescents: Benefits of mental contrasting and implementation intentions. Educational Psychology, 31(1), 17-26. https://doi.org/10.1080/01443410.2010.506003

Felver, J. C., Celis-de Hoyos, C. E., Tezanos, K., \& Singh, N. N. (2016). A systematic review of mindfulness-based interventions for youth in school settings. Mindfulness, 7(1), 34-45. https://doi. org/10.1007/s12671-015-0389-4

Greaves, C. J., Sheppard, K. E., Abraham, C., Hardeman, W., Roden, M., Evans, P. H., \& Schwarz, P. (2011). Systematic review of reviews of intervention components associated with increased effectiveness in dietary and physical activity interventions. BMC Public Health, 11(1), 1-12. https://doi. org/10.1186/1471-2458-11-119

Hölzel, B. K., Carmody, J., Vangel, M., Congleton, C., Yerramsetti, S. M., Gard, T., \& Lazar, S. W. (2011). Mindfulness practice leads to increases in regional brain gray matter density. Psychiatry Research: Neuroimaging, 191(1), 36-43. https://doi.org/10.1016/j.pscychresns.2010.08.006

Kabat-Zinn, J. (2003). Mindfulness based interventions in context: Past, present, and future. Clinical Psychology: Science and Practice, 10(2), 144- 156. https://doi.org/10.1093/clipsy.bpg016

Keng, S., Smoski, M. J., \& Robins, C. J. (2011). Effects of mindfulness on psychological health: A review of empirical studies. Clinical Psychology Review, 31(6), 1041-1056. https://doi.org/10.1016/j. cpr.2011.04.006 
Lollgen, H., Bockenhoff, A., \& Knapp, G. (2009). Physical activity and all-cause mortality: An updated meta-analysis with different intensity categories. International Journal of Sports Medicine, 30(03), 213-224. https://doi.org/10.1055/s-0028-1128150

Lentz, T. A., \& Brown, C. (2019). Mindfulness and health behaviors in college students: The moderating role of sleep. Journal of American College Health, 67(6), 505-514. https://doi.org/10.1080/07448481.2 018.1497638

Leyland, A., Rowse, G., \& Emerson, L. (2019). Experimental effects of mindfulness inductions on self-regulation: Systematic review and meta-analysis. Emotion, 19(1), 108. https://doi.org/10.1037/ emo0000425

Lyzwinski, L. N., Caffery, L., Bambling, M., \& Edirippulige, S. (2018). Relationship between mindfulness, weight, and weight-related behaviors in college students: A systematic review. Alternative \& Complementary Therapies, 24(5), 202-214. https://doi.org/10.1089/act.2018.29182.Inl

McAuley, E., Mullen, S. P., Szabo, A. N., White, S. M., Wójcicki, T. R., Mailey, E. L., . . Erickson, K. (2011). Self-regulatory processes and exercise adherence in older adults: Executive function and self-efficacy effects. American Journal of Preventive Medicine, 41(3), 284-290. https://doi.org/10.1016/j. amepre.2011.04.014

Miller, A. L., Lo, S. L., Bauer, K. W., \& Fredericks, E. M. (2020). Developmentally informed behaviour change techniques to enhance self-regulation in a health promotion context: A conceptual review. Health Psychology Review, 14(1), 116-131. https://doi.org/10.1080/17437199.2020.1718530

Neace, S. M., Hicks, A. M., DeCaro, M. S., \& Salmon, P. G. (2020). Trait mindfulness and intrinsic exercise motivation uniquely contribute to exercise self-efficacy. Journal of American College Health. https://doi.org/10.1080/07448481.2020.1748041

Penedo, F. J., \& Dahn, J. R. (2005). Exercise and well-being: A review of mental and physical health benefits associated with physical activity. Current Opinion in Psychiatry, 18(2), 189-193. https://doi. org/10.1097/00001504-200503000-00013

Rebar, A. L., Stanton, R., Geard, D., Short, C., Duncan, M. J., \& Vandelanotte, C. (2015). A meta-meta-analysis of the effect of physical activity on depression and anxiety in non-clinical adult populations. Health Psychology Review, 9(3), 366- 378. https://doi.org/10.1080/17437199.2015.1022901

Rhodes, R. E., \& Pfaeffli, L. A. (2010). Mediators of physical activity behaviour change among adult non-clinical populations: A review update. International Journal of Behavioral Nutrition and Physical Activity, 7(1), 37. http://www.ijbnpa.org/content/7/1/37.

Roberts, K. C., \& Danoff-Burg, S. (2010). Mindfulness and health behaviors: Is paying attention good for you? Journal of American College Health, 59(3), 165-173. https://doi.org/10.1080/07448481.2010. 484452

Robin, N., Toussaint, L., Sinnapah, S., Hue, O., \& Coudevylle, G. R. (2020). Beneficial influence of mindfulness training promoted by text messages on self-reported aerobic physical activity in older adults: A randomized controlled study. Journal of Aging \& Physical Activity, 28(3), 406-414. https://doi. org/10.1123/japa.2019-0002

Rothman, A. J., Baldwin, A. S., Hertel, A. W., \& Fuglestad, P. T. (2004). Disentangling behavioral initiation and behavioral maintenance. In K. Vohs, \& R. Baumeister (Eds.), Handbook of Self-Regulation. Research, Theory, and Applications. Guilford Press 130-148. Retrieved from: https://www.researchgate.net/ profile/Austin_Baldwin/publication/232574200_Self-regulation_and_behavior_change_Disentangling_behavioral_initiation_and_behavioral_maintenance/links/54ad570b0cf2213c5fe3c21f.pdf

Saelens, B. E., Gehrman, C. A., Sallis, J. F., Calfas, K. J., Sarkin, J. A., \& Caparosa, S. (2000). Use of self-management strategies in a 2-year cognitive-behavioral intervention to promote physical activity. 
Behavior Therapy, 31(2), 365-379. https://doi.org/10.1016/S0005-7894(00)80020-9

Sagui-Henson, S. J., Blevins, C. L., \& Levens, S. M. (2018). Examining the psychological and emotional mechanisms of mindfulness that reduce stress to enhance healthy behaviours. Stress \& Health: Journal of the International Society for the Investigation of Stress, 34(3). https://doi.org/10.1002/ smi.2797

Sala, M., Rochefort, C., Lui, P. P., \& Baldwin, A. S. (2019). Trait mindfulness and health behaviours: A meta-analysis. Health Psychology Review. https://doi.org/10.1080/17437199.2019

Samdal, G. B., Eide, G. E., Barth, T., Williams, G., \& Meland, E. (2017). Effective behaviour change techniques for physical activity and healthy eating in overweight and obese adults; systematic review and meta-regression analyses. International Journal of Behavioral Nutrition and Physical Activity, 14(1), 42. https://doi.org/10.1186/s12966-017-0494-y

Schneider, J., Malinowski, P., Watson, P. M., \& Lattimore, P. (2019). The role of mindfulness in physical activity: A systematic review. Obesity Reviews, 20(3), 448-463. https://doi.org/10.1111/obr.12795

Shapiro, S. L., Carlson, L. E., Astin, J. A., \& Freedman, B. (2006). Mechanisms of mindfulness. Journal of Clinical Psychology, 62(3), 373-386. 10.1002/jclp.20237

Strowger, M., Kiken, L. G., \& Ramcharran, K. (2018). Mindfulness meditation and physical activity: Evidence from 2012 national health interview survey. Health Psychology, 37(10), 924-928. https://doi. org/10.1037/hea0000656

Tang, Y., Fan, Y., Lu, Q., Tan, L., Tang, R., Kaplan, R. M., . . Reiman, E. M. (2020). Long-term physical exercise and mindfulness practice in an aging population. Frontiers in Psychology, 11, 358. https://doi. org/10.3389/fpsyg.2020.00358

Teixeira, P. J., Carraça, E. V., Marques, M. M., Rutter, H., Oppert, J., De Bourdeaudhuij, I., . . Brug, J. (2015). Successful behavior change in obesity interventions in adults: A systematic review of self-regulation mediators. BMC Medicine, 13(1), 84. https://doi.org/10.1186/s12916-015-0323-6

Tsafou, K., De Ridder, Denise T. D., van Ee, R., \& Lacroix, J. P. W. (2016). Mindfulness and satisfaction in physical activity: A cross-sectional study in the dutch population. Journal of Health Psychology, 21(9), 1817-1827. https://doi.org/10.1177/1359105314567207

Tsafou, K., Lacroix, J. P., van Ee, R., Vinkers, C. D., \& De Ridder, D. T. D. (2017). The relation of trait and state mindfulness with satisfaction and physical activity: A cross-sectional study in 305 dutch participants. Journal of Health Psychology, 22(10), 1221-1232. https://doi. org/10.1177/1359105315624748

Warburton, D. E., Nicol, C. W., \& Bredin, S. S. (2006). Health benefits of physical activity: the evidence. CMAJ, 174(6), 801-809. https://doi.org/10.1503/cmaj.051351

World Health Organization. (2013) Physical inactivity: A global public health problem. Global Strategy on Diet, Physical Activity and Health. Retrieved from: https://www.who.int/dietphysicalactivity/factsheet_inactivity/en/

World Health Organization. (2010). Prevalence of insufficient physical activity. Retrieved from: https:// www.who.int/gho/ncd/risk_factors/physical_activity_text/en/

Yang, C., \& Conroy, D. E. (2019). Mindfulness and physical activity: A systematic review and hierarchical model of mindfulness. International Journal of Sport and Exercise Psychology, 1-24. https://doi.org /10.1080/1612197X.2019.1611901 